\title{
Correspondence
}

\section{Pancuronium bromide induced joint contractures in the newborn}

Sir,

I read with interest the paper of Sinha and Levene ${ }^{1}$ reporting the development of contractures in three of 13 ventilated infants treated with pancuronium bromide, as this is at such variance with the experience of the neonatal intensive care unit in Cambridge. In the past three years we have paralysed 85 of a total of 246 ventilated infants and none of these have developed contractures. This difference is difficult to explain. Although the development of contractures after the use of muscle relaxants is to some extent a dose related effect, ${ }^{2}$ this is not the answer as we have used the same dose regimen as decribed by Sinha. ${ }^{1}$ Similarly, many of our infants were also subject to the possible potentiation of pancuronium's action by simultaneous gentamicin administration. ${ }^{3}$ Differences in maturity may be a possible explanation as our population of ventilated infants were slightly more immature (mean (SD), $29.2(2.7)$ weeks) than the Leicester infants (mean (SD), $34.5(5.0)$ weeks). Although the published reports suggest a vulnerable period for joint development, this remains somewhat controversial; data from animal experiments suggest that older fetuses seem at greater risk of developing contractures after prolonged immobilisation ${ }^{2}$ whereas reports of human neonates so affected are of fetuses exposed to possible pancuronium immobilisation in the first trimester of pregnancy. ${ }^{4}$ Indeed, the Leicester data showed contractures developing after pancuronium administration in one preterm, one term, and one postterm infant.

Theoretically, the risk of developing contractures should increase with an increase in the time of prolonged immobilisation. Yet in the Leicester data one infant was paralysed for only 6 hours and developed contractures and one other for only two days. In the study we have recently reported, using pancuronium selectively to prevent pneumothoraces, ${ }^{5}$ pancuronium was given to the trial infants for a similar time span (mean (SD), $3 \cdot 5(1 \cdot 2)$ days) as the Leicester babies (mean (SD), $2 \cdot 5(2 \cdot 0)$ days) and our trial infants were given a similar number of doses (mean (SD), 9.6 (5.4)) as the Leicester babies (mean (SD), 7.8 $(10 \cdot 6)$ ), suggesting that the duration of paralysis with pancuronium did not explain the discrepancy in the two studies.

Pancuronium is a very useful drug preventing serious complications of mechanical ventilation. ${ }^{5}$ The Leicester report of infants developing contractures after its use is worrying but perhaps their group of ventilated babies was not typical; at least one of their infants was abnormal before the drug was given. We have not experienced this problem yet have used pancuronium in a large group of babies and are unable to explain the contractures developing in the Leicester babies by differences in drug usage.
Perhaps the answer lies with our nursing staff who should be commended for their excellent care of these possibly 'at risk' babies which prevents this problem occurring.

\section{References}

' Sinha SK, Levene MI. Pancuronium bromide induced joint contractures in the newborn. Arch Dis Child 1984:59:73-5.

2 Drachman DB, Coulombre AJ. Experimental club foot and arthrogryphosis multiplex congenita. Lancet 1962;ii:523-6.

${ }^{3}$ Warner WA. Sanders E. Neuromuscular blockade associated with gentamicin therapy. JAMA 1971:215:1153-4.

4 Vago RH. Arthrogryphosis following treatment of maternal tetanus with muscle relaxant. Arch Dis Child 1970;45:277-9.

'Greenough A, Wood S, Morley CJ, Davies JA. Pancuronium prevents pneumothoraces in ventilated premature babies who actively expire against positive pressure inflation. Lancet $1984 ; \mathbf{i}: 1-3$.

\section{Anne Greenough University of Cambridge Clinical School, Addenbrooke's Hospital, Hills $R d$, \\ Cambridge CB2 $2 Q Q$}

\section{Maldescent of the testis}

\section{Sir,}

I read with interest and approval the annotation by Professor Lewis Spitz regarding maldescent of the testis ${ }^{1}$ but although accepting that orchidopexy from the age of 2 years is probably desirable, I think that in practice it ill behoves paediatric surgeons to advocate this for many orchidopexies are done by general surgeons with an 'interest' in paediatric surgery. I have little doubt that many of these are competent gentlemen but I also have little doubt that very often an orchidopexy is delegated to a junior member of staff. If I find an orchidopexy in a patient of 2 years difficult I am quite certain that a general surgeon would find this even more so. Hence I think there is a great danger that this doctrine if practised too widely will lead to more testes atrophying from mishandling of the delicate blood vessels of the cord.

The other point that needs to be made is that many of the undescended testes are picked up at routine school examination at the age of 4 or 5 years. This would still seem to be the most likely optimum time for surgery in view of the screening that goes on at this age. Paediatricians cannot always be relied upon to refer all patients with undescended testes at an early age and indeed sadly it is not unusual for children under the age of 4 years to be admitted to a medical ward for various problems and for no comment to be made about their testicular state! I believe that we as paediatricians and paediatric surgeons must put our own house in order before advocating a widespread policy of orchidopexy at age 2 which might lead to greater devastation than that intended. 